\title{
Aunando esfuerzos: Grupos multifamiliares en el abordaje de los trastornos de la alimentación
}

\section{Joining forces: Multi-family groups in the approach of eating disorders}

\author{
M $^{\mathrm{a}}$ Pilar Vilariño Besteiro ${ }^{(1)}$, Belén Unzeta Conde ${ }^{(1)}$, \\ Teodoro Uría Rivera ${ }^{(2)}$, César Pérez Franco ${ }^{(2)}$, Enrique Guerra Gómez ${ }^{(1)}$ \\ (1) UTCA Hospital Universitario Santa Cristina, España \\ (2) Hospital Doce de Octubre, España
}

\begin{abstract}
Resumen: En este artículo revisamos el concepto de grupo multifamiliar, su desarrollo histórico, los beneficios y objetivos de esta intervención, la función del terapeuta en estos grupos y algunos aspectos técnicos. También revisamos algunas investigaciones acerca de su aplicación en los Trastornos de la Conducta Alimentaria (TCA). Finalmente describimos nuestra experiencia en la Unidad de Trastornos de la Conducta Alimentaria del Hospital Universitario Santa Cristina de Madrid explicando tanto la metodología de trabajo como los temas abordados en los grupos, y compartimos algunas de las impresiones de nuestros pacientes y sus familiares al respecto. Si bien este tipo de intervención tiene mucha potencialidad terapéutica, no debe usarse como única modalidad de tratamiento en los TCA.
\end{abstract}

Palabras clave: trastornos alimentarios, grupos multifamiliares, intervención familiar.

Abstract: In this article we review the concept of multifamily group, its historical development, the benefits and objectives of this intervention, the role of the therapist in these groups and some technical aspects. We also review some research about its application in the Eating Disorder (ED). Finally, we describe our experience in the Unit of Eating Disorders at the Santa Cristina University Hospital in Madrid, explaining both the work methodology and the issues addressed in the groups and we share some of the impressions of our patients and their families about it. Although this type of intervention has great therapeutic potential, it should not be used as the only treatment modality in eating disorders.

Keywords: eating disorders, multifamily groups, family intervention.

\footnotetext{
Ma Pilar Vilariño Besteiro es Psicóloga Clínica, UTCA Hospital Universitario Santa Cristina de Madrid.

Belén Unzeta Conde es Psiquiatra,UTCA Hospital Universitario Santa Cristina de Madrid.

Teodoro Uría Rivera es Psiquiatra, Hospital Doce de Octubre de Madrid.

César Pérez Franco es Terapeuta Ocupacional, UTCA Hospital Doce de Octubre de Madrid.

Enrique Guerra Gómez es Psiquiatra Coordinador, UTCA Hospital Universitario Santa Cristina de Madrid.
}

La correspondencia sobre este artículo debe enviarse a la primera autora al email: pvilarinob@yahoo.es

(cc) BY-NC-ND Este es un artículo Open Access bajo la licencia CC BY-NC-ND. 


\section{Grupos Multifamiliares. Desarrollo Histórico}

El termino Terapia Multifamiliar es confuso porque engloba una pluralidad de intervenciones cuyo denominador común es reunir a un grupo de familias en un mismo espacio terapéutico pero que pueden ser conducidas desde orientaciones teóricas distintas. Los grupos multifamilares pueden variar en cuanto a su estructura, frecuencia y duración de sesiones, número de participantes, tipo de problemáticas atendidas, metas, roles de los terapeutas, técnicas utilizadas, entre otros aspectos (Sempere y Fuenzalida, 2017). Edwards (2001) ha enumerado hasta 16 orientaciones teóricas en este tipo de intervención.

Mascaró (2010) entiende el grupo multifamiliar como una representación de una microsociedad de familias de diferentes orígenes que participan en una experiencia común donde compartir su sufrimiento, confrontar sus dificultades y ser contenidas y sostenidas en el tiempo. Su realización requiere integrar conocimientos del funcionamiento psicológico individual, de la dinámica de la familia, de los grupos pequeños y de las relaciones e influencias sociales.

El grupo multifamiliar hace referencia al setting en el que pueden desarrollarse procesos terapéuticos concomitantes (...) con elementos comunes basados en la teoría de los grupos, de los sistemas familiares, y en la que la terapia grupal actúa como engranaje que potencia los procesos de cambio tanto a nivel individual como a nivel de las dinámicas familiares. (Sempere y Fuenzalida, 2017, p. 30).

Para desarrollar la perspectiva histórica de los grupos multifamiliares tomamos como base las aportaciones de Sempere y Fuenzalida $(2013,2017)$. Estos autores plantean que la terapia multifamiliar ha tenido tres ejes de difusión que desarrollaremos a continuación: Argentina, EE.UU. y Europa occidental.

En EE.UU., en el año 1953 Abrahams y Varon realizan un grupo de carácter observacional de las interacciones simbióticas entre madres e hijos psicóticos. Pero el origen y la denominación de intervención multifamiliar se remontan a la década de los 60 de la mano de Peter Laqueur (1976). Este autor utiliza inicialmente la denominación de Terapia Grupal para Múltiples Familias para referirse a una adaptación de la técnica de grupo al tratamiento de familias de pacientes psicóticos y observó que el uso de estos grupos reducía hasta un $80 \%$ las readmisiones en el hospital, que producía cambios más rápidos que las intervenciones individuales y que era un método eficiente en cuanto a tiempo y coste.

El recopilador de la obra de Laqueur fue Lewis Foster desde un paradigma sistémico basado en la escuela estratégica y en la teoría de sistemas. Este autor parte de la base de que si queremos ver cómo funciona un sistema, tenemos que ver lo que hace y no lo que dice que hace (Foster, 1991).

Otro pionero en la aplicación de intervenciones multifamiliares desde un planteamiento psicoeducativo, estructurado y con efectividad probada es McFarlane (2002). Su planteamiento establece cuatro tipos de intervención: autotriangulación del terapeuta, interpretación grupal, enlace transfamiliar (los miembros de una familia comentan acerca de lo observado en otra), y el tratamiento interfamiliar recurriendo a técnicas estructurales o estratégicas.

Por su parte, Bowen (2014) trabaja desde una perspectiva multigeneracional, transgeneracional o intergeneracional y establece como meta el incrementar el nivel de diferenciación de cada miembro de la familia. Utiliza la denominación de Terapia Familiar Múltiple o Terapia de Red con Familias Múltiples.

Otras aportaciones americanas a la intervención multifamiliar serían: Steinglass (1998) con personas con enfermedad crónica, Kaufman (1977) en el contexto de las adicciones, y el modelo Coliseum aportado por Saunders (1990) que apuesta por la horizontalidad y apertura de los profesionales, animándoles a participar y a incluir a sus propias familias, y dan un peso importante al abordaje de los secretos familiares.

En Argentina, ante la insatisfacción con los abordajes tradicionales, en la década de los 60 Jorge García Badaracco pone en marcha en el Hospital Borda de Buenos Aires, grupos multifamiliares donde intervenían terapeutas, familias, pacientes y enfermería (Mascaró, 2010). La denominación que utiliza este autor es el de Psicoanálisis multifamiliar en el que combina aportaciones sistémicas, psicoanalíticas y del modelo de comunidad terapéutica.

Los grupos multifamiliares, según García Badaracco (2000), permiten integrar en un solo abordaje diversos recursos terapéuticos y trabajar simultáneamente la dimensión individual, familiar y social de la mente. Considera que los pacientes se curan cuando son vistos como personas y no como pacientes. 
Un concepto básico en la teorización de García Badaracco es el de interdependencia recíproca que hace referencia a las pautas relacionales sobre las que se construyen las identificaciones que configuran la identidad del ser humano. Estas interdependencias pueden ser normogénicas (si favorecen el desarrollo y el crecimiento del yo: virtualidad sana) o patógenas si impiden el desarrollo de los recursos yoicos (Sempere y Fuenzalida, 2017).

El psicoanálisis multifamiliar funciona como una mente ampliada cuya meta consiste en ayudar a los diferentes miembros a liberarse de interdependencias patógenas y a potenciar su virtualidad sana (García Badaracco, 1989, 2000). Esta modalidad de intervención se difunde por Europa y en España comienza su andadura en las décadas de los 80 y 90 de la mano de Ayerra y López (2003) y Sempere (2012) entre otros.

Dentro del continente europeo se desarrollan otras intervenciones de carácter multifamiliar:

- Cooklin, Miller y McHung (1983) trabajan desde un paradigma que integra el modelo sistémico (estructural y de la Escuela de Milán), el de comunidad terapéutica, la antipsiquiatría y la terapia social, con familias que conviven durante 5 días a la semana en un hospital de día.

- Asen (2006), en Reino Unido, plantea que en la terapia multifamiliar coexisten una mezcla de prácticas psicodinámicas, de terapia de grupo, de terapia familiar y de la teoría del apego y de la mentalización.

En España, Alcalde y Montero (2014) consideran que el grupo multifamiliar es otra manera de responder a las necesidades de los usuarios y familiares, de mejorar la red de apoyo, la comunicación, lograr normalización de roles y tener apoyo emocional, que les permita avanzar en su proceso terapéutico. Además es un lugar de aprendizaje en el que unas familias sirven de modelos a otras para facilitar la adquisición de actitudes y formas de comunicación más positivas, con menor criticismo y hostilidad.

En el denominado Foro de Elche, y de la mano de Javier Sempere entre otros, se ha desarrollado el modelo de Terapia Interfamiliar que es un modo de intervención ecléctico e integrador de los diferentes modelos de intervención multifamiliar. Sus pilares serían el psicoanálisis multifamiliar, la teoría del apego y las prácticas dialógicas en red.

La terapia interfamiliar favorece la interacción comunicativa analógica y verbal entre los participantes - profesionales y no profesionales - creando una compleja matriz grupal transgeneracional, multicultural y multidisciplinaria en la cual se teje un multiverso en varis planos: individual, intrafamiliar, interfamiliar y social. Los aspectos biológicos, cognitivos, intrapsíquicos, psicodinámicos, vinculares y sistémicos -entre otros- son tenidos en cuenta en el mismo grado, entendiendo así que no hay verdades absolutas y que estamos sometidos a múltiples determinantes culturales y sociológicos que se reflejan en el arcoíris multifamiliar (Sempere y Fuenzalida, 2017, p. 90 ).

\section{Principios Fundamentales del Modelo Interfamiliar}

Según Sempere y Fuenzalida $(2013,2017)$ el modelo Multifamiliar se nutre de diferentes teorías y modelos terapéuticos.

Teoría del apego. Se incorpora la importancia de los vínculos, el impacto negativo de la falta de estos, y la importancia de una base segura en el tratamiento, entre otras aportaciones.

Terapia familiar sistémica. Este modelo se asienta en la Teoría General de Sistemas de Von Bertalanffy (1976) (concepto de familia como sistema) y en la Teoría de la Comunicación humana de Watzlaswick, Beavin, Jackson (1995). Del planteamiento sistémico se incluyen conceptos como paciente designado, roles, triangulaciones, alianzas y coaliciones, pseudomutualidad (se sacrifican los intereses individuales en aras de la supervivencia del grupo o de la familia, se prohíbe la diversidad, el debate o el conflicto), individuación y diferenciación (capacidad de separarse de la familia de origen), las lealtades invisibles, juego familiar (transacciones patológicas, rígidas y crónicas), el reencuadre o redefinición del problema, trabajo con límites y fronteras, inclusión en el contexto terapéutico de figuras significativas, estrategias narrativas como el cuestionamiento del síntoma redefiniéndolo como un acto de protección a la familia, y el cuestionamiento de la estructura y de la realidad familiar.

Terapia grupal. Desde esta perspectiva se incluyen las aportaciones de diferentes autores que se describen a continuación. 
Terapia interpersonal de Harry Sullivan (1953). De esta modalidad terapéutica se adopta la importancia de los procesos interpersonales y el cambio basado en la interacción, la retroalimentación y el aprendizaje interpersonal en el aquí y ahora del grupo.

Grupoanálisis de Foulkes (1984). Se incorporan los conceptos de matriz grupal (red de comunicaciones interpersonal, transpersonal y suprapersonal en la que toma preeminencia el inconsciente social y donde el yo es entendido como el nosotros en mí), el concepto de red o entramado de líneas de comunicación, el de plexo o red dinámica íntima y el de mirroring (los miembros del grupo se reflejan emocionalmente de forma mutua entre sí).

Grupo grande de Patrick de Maré (1988). También otros grupos de orientación dinámica como el de Bion (1985), y el grupo operativo de Pichón Riviere (1985).

Intervención en red social. Desde este modelo se incluyen diversos aspectos:

- Importancia de ser mirado y reconocido.

- El papel del aprendizaje social: la identidad social, los estereotipos y prejuicios, sesgo de correspondencia, efecto actor-observador, sesgo hacia lo negativo, efecto de congruencia con el estado de ánimo en el recuerdo de la información, ilusión de homogeneidad, y la hipótesis de contacto para reducir el prejuicio.

- El apoyo social y su impacto en la salud física y mental.

- Prácticas dialógicas y colaborativas del construccionismo social de Gergen (1973), las terapias narrativas de McNamee y Gergen (1996), que plantean que es la sociedad la que construye la lente a través de la cual miran el mundo sus miembros y la importancia del lenguaje en la creación de la realidad, y el diálogo abierto de Seikkula (1994) que incluye al paciente en las discusiones sobre su proceso terapéutico.

Neurobiología interpersonal. Considera el cerebro humano como un órgano social. Las conexiones cerebrales entre los individuos participantes en las interacciones se modelan mutuamente, de ahí el potencial de la psicoterapia grupal y su utilidad para la reparación y la reorganización de la memoria implícita.

\section{Objetivos y Beneficios del Grupo Multifamiliar}

El objetivo de los grupos multifamiliares, en base a las aportaciones de diferentes autores, podría resumirse en lo siguiente: crear un clima emocional de seguridad, libre de juicio, que sirva de sostén para compartir el sufrimiento, expresar lo silenciado y liberar a las personas de interdependencias patógenas, para que aflore su virtualidad sana; desarrollando habilidades y patrones adaptativos donde se exprese la autenticidad de cada cual, favoreciendo así el desarrollo, el crecimiento personal y el proceso de autonomía y de individuación. Para favorecer este proceso es importante el enriquecimiento de la vida de los padres para que puedan dejar volar a sus hijos más fácilmente e indagar si detrás de las actitudes sobreprotectoras existe una percepción de insuficiencia del hijo. (Ayerra y López, 2003; Brinchmann et al., 2017; Burguillo, 2010; Canzio y Zurkirch, 2015; García Badaracco, 2000; Mascaró, 2007; Pérez, Jiménez, Torregrosa y Sempere, 2016; Sempere y Fuenzalida, 2017).

Los beneficios de los grupos multifamiliares, realizando una tarea de síntesis de diversas publicaciones, serían los siguientes: favorecen la socialización, el aprendizaje de nuevos roles, permiten adquirir nuevas narrativas hacia los problemas, el aprendizaje vivencial y vicario y las actitudes de respeto, empatía y tolerancia al malestar. Estos grupos también invitan a la expresión sincera y a la autenticidad, mejoran la capacidad de mentalización, facilitan la coordinación entre terapeutas, pacientes y familias, permiten producir cambios profundos al incluir diferentes enfoques psicoterapéuticos en las mismas coordenadas espaciotemporales, posibilita las experiencias emocionales correctivas dando nuevas soluciones a situaciones pasadas, ayudan a mejorar las relaciones afectivas y a liberarse de interdependencias patógenas, contribuyen a amortiguar posturas radicales, hablar de lo silenciado y a expresar culpas y reproches brindando la posibilidad de reparar (Ayerra y López, 2003; Blajakis y López Atienza, 2002; Burguillo, 2010; Conner et al, 1998; Fortunato, Ribeiro, Lobo, Figueiredo y Cavalcanti, 2011; Martínez y Perles, 2010; Mascaró, 2007; Pérez et al, 2016; Uría, Ahijado, Serrano, Mira y Rubio, 2016).

Según Asen $(2002,2006)$ la terapia multifamiliar permite la creación de un clima de solidaridad, reduciendo el estigma y el aislamiento social, estimulando la adopción de nuevas perspectivas, aprendiendo de los otros 
y viéndonos reflejados en el espejo del otro y usando positivamente la presión grupal, el apoyo mutuo y feedback. También permite descubrir y desarrollar competencias y nuevos comportamientos en un lugar seguro, intensificar experiencias e interacciones aumentando la posibilidad de éxito y de esperanza, fortalecer la función reflexiva, la apertura, la autoconfianza, la autorreflexión y la autoestima a través del intercambio mutuo.

Además, este tipo de intervención también incluye las ventajas de la terapia de grupo en general que fueron descritas por Yalom (1995): la inspiración de esperanza, catarsis, autorrevelación, orientación, universalidad, aceptación y pertenencia, aprendizaje vicario, y por acción interpersonal, el autoentendimiento/autoconocimiento y el altruismo.

\section{Funciones del Terapeuta en el Grupo Multifamiliar}

Según Sempere y Fuenzalida (2017), la meta de la conducción del grupo multifamiliar estriba en crear un clima emocional seguro en el que los participantes encuentren un lugar donde quitarse las máscaras que los protegen de las respuestas hirientes por parte de otras personas y se conecten íntimamente con sus propias vidas así como con los procesos psicológicos de los otros. El fin último es facilitar que los participantes realicen su proceso terapéutico a través de restaurar sus relaciones intra y extrafamiliares. Para estos autores, los objetivos de la conducción del grupo serían favorecer la cohesión grupal, potenciar el dialogo abierto, y polifónico, lograr el apoyo emocional entre los participantes y promover la flexibilidad en la comunicación grupal, la sorpresa y la espontaneidad.

Tratando de integrar los planteamientos de diferentes autores, las funciones que debe realizar un terapeuta conductor de grupos multifamiliares serían las siguientes:

- Ayudar a crear un clima de seguridad y contención emocional que favorezca la expresión emocional y el compromiso con la tarea, evitando la intelectualización, tratando de bajar de la metáfora al contenido concreto (Pichón-Riviere, 1985; Ayerra y López, 2003), favoreciendo la expresión de los resentimientos, culpas, envidias y odios. Observar la pertinencia de lo expresado -ver si es para taponar verdades o es para elaborar contenidos- y favorecer y apoyar la multiplicidad de transferencias (Martínez y Perles, 2010).

- Escuchar y favorecer la escucha, que se hable desde la propia vivencia (Burguillo, 2010), más desde el yo que desde el tu, privilegiando la experiencia frente a la explicación (Sempere y Fuenzalida, 2017), evitando los juicios de valor y favoreciendo el diálogo entre los participantes (Ayerra y López, 2003). Es mejor que sean otros miembros que el conductor del grupo, los que sugieran cambios en base a su propia experiencia (Pérez et al, 2016).

- Ayudar a desgranar el discurso del paciente del que sostienen los otros significativos, resaltando aquellas palabras que tienen un significado especial para éste (Ayerra y López, 2003); señalar interdependencias patógenas (Arroyo Guillamón, Fernández-Mayoralas, Reguera Nieto y Martínez Pérez, 2014; García Badaraco, 1989), ver las demandas de atención y afecto, y la necesidad de que les den un lugar en su mente y que respeten el derecho a ser diferente, que se oculta tras las conductas agresivas. (Ayerra y López, 2003; Blajakis y López, 2002). Acompañar a aquellos pacientes que tienen mayor fragilidad yoica incluso con contacto físico (Ayerra y López, 2003).

- Validar y acompañar en la expresión de emociones para lograr una experiencia emocional correctiva necesaria para el cambio terapéutico. También es importante ayudar a buscar paralelismos entre las situaciones del aquí y ahora y vivencias pasadas. No hay que evitar temas emocionalmente difíciles y hay que proporcionar apoyo ante la expresión de contenidos comprometidos (Pérez et al, 2016).

- Poner sobre la mesa los emergentes grupales (Pichón Riviere, 1985); saber leer el clima del grupo y desplegar recursos en función de las necesidades de éste. Por esta razón, es necesaria la capacidad de improvisación (Brichmannn et al, 2017). Respetar el tiempo que cada persona tiene para participar (García Badaracco, 1989).

- Hay que atender al contenido, pero sobre todo a las interacciones, y tener capacidad de resumir, de poner orden y de reconducir el grupo, favoreciendo que el contenido sea aplicable a todos, tratando de encontrar semejanzas entre los participantes (Brichmannn et al, 2017; Pérez et al., 2016). 
- Alentar, favorecer que asuman la posibilidad y la responsabilidad del cambio (Ramos y de Miguel, 2017). - Es necesario atender a los individuos, a las familias y al grupo en general (visión de conjunto). Saber utilizar un tono correcto en el lugar adecuado y en el momento correcto, y también usar de forma adecuada el sentido del humor. Es importante que cada terapeuta encuentre su propio estilo e individualidad y su propio modo de hacer las cosas sin tratar de imitar a otros (Brichmannn et al, 2017).

- Señalar comportamientos y relaciones personales (interdependencias) tratando de rescatar los aspectos positivos, las demandas emocionales y las necesidades profundas que se esconden tras una relación problemática (Mascaró, 2007). También es importante explorar duelos no resueltos que a veces se expresan de forma simbólica (Ayerra y López, 2003).

- El terapeuta no debe tomarse como personal lo sucedido en el grupo. Debe mantener una actitud no directiva, exploratoria, mostrando una curiosidad activa y un interés genuino, manteniendo la autoridad y el poder sin ejercerlo de modo tradicional pero interviniendo en casos necesarios - más al inicio del grupo o para rescatar a algunos miembros si la emoción expresada es muy alta, para limitar a los que pueden acaparar en exceso el grupo, para proteger a un paciente confuso de la sobreestimulación o para interceder en escaladas simétricas-. Se trata de adoptar una postura flexible, pivotando desde posiciones más distendidas a momentos de mayor profundidad o trascendencia (Sempere y Fuenzalida, 2017).El conductor actúa como un mero catalizador de la conversación entre participantes, convirtiéndose en un arquitecto del diálogo grupal y en un modo de observador participante, atendiendo a lo verbal y no verbal, y colaborando en hacer explícito lo implícito de las narrativas (Sempere y Fuenzalida, 2017). Asimismo, el terapeuta debe cuidarse de no asumir la posición de omnipotencia en la que el grupo le quiere colocar (Ayerra y López, 2003), y debe sostener una actitud más de querer aprender que de mostrarse como experto (García Badaracco, 1989). No se trata de decir lo que tiene que hacer sino ayudar a que lo descubra por sí mismo (Rienecke, 2017).

Basándonos en los planteamientos de diversos autores, las cualidades personales que requiere el terapeuta del grupo multifamiliar serían: la humildad, prudencia, actitudes interpretativas, no querer tener razón, empatía, tolerancia al sufrimiento, a las proyecciones, a la expresión emocional y a la incertidumbre. Saber mostrarse compasivo sin ser crítico u hostil, ni manipular a los pacientes o las familias, actitud de disponibilidad, autenticidad, adecuación, carisma, fuerza y seguridad. Así mismo, el terapeuta debe tener sentido de responsabilidad y de respeto, confianza en el grupo y en las personas, capacidad de interactuar con otros terapeutas y estar dispuesto a aprender de los pacientes y de las familias (Brichmannn et al, 2017; Canzio y Zurkirc, 2015; García Badaracco, 1989; Mascaró 2007; Sempere y Fuenzalida, 2017).

En un estudio de Gulliksen, Espeset, Nordbo y Holte (2012) las características de los terapeutas más valoradas por pacientes con anoréxica nerviosa (AN) son la vitalidad y el humor.

\section{Algunos Aspectos Técnicos}

Ayerra y López (2003) resaltan la importancia de una buena coordinación entre los miembros del equipo y que compartan por parte de los integrantes del mismo un mismo modelo de comprensión de la salud y la enfermedad mental. También subrayan la necesidad de realizar un post-grupo donde se intercambien las impresiones sobre el mismo, pero siguiendo la máxima de hablar con las personas en lugar de hablar de las personas, es decir, poder devolver al paciente/familia las reflexiones a las que se ha llegado.

Brinchmann, et al., (2017) consideran que en el grupo multifamiliar no se trata tanto de hablar acerca de un tema en concreto sino más bien saber leer por dónde va el grupo y tratar de regular la temperatura del mismo, que no debe ser ni muy alta ni muy baja; se trata de lograr suficiente impulso, tensión, desafío, inquietud y pequeñas crisis de tal manera que siempre haya material para trabajar. Estas crisis deben abordarse, y poner sobre la mesa, tanto los temas difíciles como las observaciones que se realizan. Es importante saber realizar preguntas que ayuden a mantener ese equilibrio entre la relajación y la construcción o elaboración.

Algunas herramientas útiles para dinamizar el grupo según las recomendaciones de Sempere y Fuenzalida (2017) serían: 
- Centrarse en el aquí y ahora de las interacciones que suceden en el grupo, buscado analogías extragrupales, y sin excluir abordar aspectos del pasado o trangeneracionales.

- Uso de la trasparencia del conductor contratransferencia elaborada o enacment) y la metacomunicación terapéutica: hablar de cómo se siente el terapeuta ante lo que aportan los participantes y no sobre lo que hacen.

- Privilegiar la experiencia frente a la explicación, todo cambio terapéutico exige una experiencia emocional correctiva, para lo cual es necesario movilizar las emociones y afecto, seguido del análisis e integración de los mismos. Por eso es fundamental ser capaz de tolerar la expresión emocional dentro del grupo.

- Mantener una actitud empática, genuina, y de cuestionamiento y neutralidad, evitando generalizaciones, animando a expresar pensamientos y emociones, promoviendo el feedback y devolviendo las preguntas al grupo para favorecer la comunicación directa entre los miembros.

- Evitar los diagnósticos y los tecnicismos, usando un lenguaje claro y conciso, no cruel pero llamando a las cosas por su nombre. Es importante que el terapeuta también reconozca sus errores, y tener en cuenta que el acto terapéutico va más allá de las palabras (gestos, miradas...).

- Promover el sentimiento de pertenencia al grupo (uso del nosotros, presentar a los nuevos, hacer referencia a los ausentes, recordar aniversarios o acontecimientos importantes, etc.).

- Entender la resistencia como una autoprotección y ayudar a indagar la funcionalidad del síntoma; dirigirnos al potencial sano de la persona y mantener una postura de optimismo respecto al potencial de cambio.

- Uso de la connotación positiva, la mentalización, la poesía, el material de los sueños, la metáfora y el humor de forma adecuada, y abordar temas existenciales como el sentido de la vida, la muerte, la libertad o los deseos.

- También puede recurrirse a recursos técnicos: textos, películas, psicodrama, etc.

- Definir el rol de los terapeutas como compañeros de viaje en el proceso (todos en el mismo barco), éstos pueden llevar al grupo más allá de los límites a los que él ha llegado (el sanador herido).

- Ayudarles a asumir la responsabilidad sobre lo que les ocurre como primer paso para poder cambiar algo y favorecer la toma de decisiones como acción sanadora.

\section{Grupos Multifamiliares en TCA}

Las aplicaciones de la terapia multifamiliar en los TCA comenzaron a finales de la década de los 80, algunas experiencias pioneras fueron las de Slagerman y Yager (1989) y las de Wooley y Lewis (1987).

La evidencia respecto a la intervención multifamiliar en TCA es limitada. Muchos estudios son descriptivos, con muestras pequeñas, o basados en la satisfacción de pacientes y familiares. Aunque no existe un modelo de grupo multifamiliar que se considere tratamiento basado en la evidencia, la investigación arroja resultados prometedores respecto a la reducción de síntomas, mejoras en la calidad de vida, en la autoestima y el estado de ánimo (tanto en el paciente como en los padres), en la autoeficacia parental y respecto a la carga del cuidador. Además, se observan altos niveles de satisfacción con el tratamiento, bajas tasas de abandono, de readmisión hospitalaria y menor tiempo de ingreso, mejora de la comunicación familiar, menos disputas relacionadas con la comida y mejor ambiente en casa (Gelin, Cook-Darzens, Simon y Hendrick , 2015).

La adaptación de los grupos multifamiliares para niños y adolescentes con TCA ha sido desarrollada por dos grupos de trabajo en un contexto de hospital de día, ambos toman como referencia la Terapia Basada en la Familia del Hospital de Maudsley (Lock, Le Grang, Agras y Dare, 2001). Por un lado, en Reino Unido, Dare y Eisler (2000) llevan a cabo un modelo de intervención multifamiliar con adolescentes diagnosticados de anorexia nerviosa (AN) y bulimia (BN). Se trata de un programa estructurado, focalizado en la mejora de la sintomatológica, y con un componente psicoeducativo. En sus conclusiones refieren una mejoría clínica en los pacientes tratados, una reducción del clima de disputa y tensión familiar, un aumento del clima de cooperación y apoyo, así como una reducción del número de abandonos y un aumento del nivel de satisfacción de pacientes y familias con el tratamiento. 
Por otro lado, Scholz y Asen (2001) refieren una experiencia llevada a cabo en el Servicio de Psiquiatría para adolescentes con TCA en Dresde, Alemania. En este caso el objetivo fundamental de la intervención multifamiliar se centra en restaurar y fortalecer las jerarquías de los padres, incrementar la cooperación, ofrecer apoyo, reducir el aislamiento familiar y favorecer un clima de solidaridad. Los padres acuden todo el día, durante una semana y/o fines de semana, al tratamiento, y participan en comidas conjuntas, encuentros formales e informales, debates, trabajos de arte creativo y salidas. Entre los beneficios de estos grupos se encuentran la reducción de la estigmatización, el aprendizaje y el apoyo mutuo, la retroalimentación, el favorecer la participación activa de los padres, experimentar con la crianza en sustitución (los distintos padres con los diferentes hijos), neutralizar las relaciones crónicas personal-paciente, crear el efecto que denominan "hot house" e inducir esperanza. Como conclusiones de su experiencia con los grupos multifamiliares señalan que estos grupos favorecen la reducción de los síntomas, disminuyen las recaídas y las readmisiones hospitalarias. También facilitan una recuperación más rápida tras la recaída, dado que la familia tiene más capacidad de afrontar situaciones críticas y pueden usar la ayuda de forma apropiada, y permiten un trabajo más intensivo y efectivo con problemas familiares y conflictos.

En los años 90, en el Hospital Robert Debre de París, Cook-Darzens (2014) desarrolla otro modelo de tratamiento multifamiliar sistémico e integrador para adolescentes con TCA con varios tratamientos fallidos. Este modelo combina la perspectiva sistémica, la terapia familiar médica, la terapia basada en la familia de Maudsley y el Nuevo Método Maudsley (Treassure, Smith y Crane (2007). Las metas de este tipo de intervención serían: superar los sentimientos de aislamiento emocional/social y la estigmatización, crear un clima de solidaridad, confianza y apoyo mutuo, comprender el TCA y su funcionalidad, lograr un sentido de dominio y esperanza, ayudar a los padres a asumir la responsabilidad y a reorganizar la vida familiar y las pautas relacionales, apoyar a las familias en el proceso de cambio favoreciendo la autorreflexión acerca de su identidad presente, pasada y futura y sobre lo que deben cambiar y lo que deben mantener. Además permiten revisar roles, rutinas, valores, creencias y actividades, fortalecer la autoestima y el aprendizaje a través del reflejo en los demás, favorecer la ayuda a los otros, estimular la esperanza y el optimismo, y mejorar la cooperación entre las familias y el equipo. Durante el grupo se diferencian fases distintas, inicialmente se trata de crear un clima de confianza, cohesión y una base segura. Durante esta fase inicial los temas están más centrados en el TCA pero poco a poco se abordan temas más generales relacionados con la identidad familiar y adolescente, la separación-individuación y por último y de modo gradual, se produce la terminación del grupo.

Gelin, Cook-Danzers et al. (2015) observan que la intervención multifamiliar produce una mejora del IMC, de los síntomas físicos y psicológicos del TCA, en el nivel de angustia y en la calidad de vida en adolescentes. Por otro lado, Stewart, Voulgari, Eisler, Hunt y Simic (2015) observan una mejoría en la clínica de TCA, en las habilidades de afrontamiento y en la depresión en adolescentes con bulimia (BN) utilizando un programa de intervención multifamiliar basado en el modelo Maudsley.

Colahan y Robinson (2002) combinan técnicas experienciales, creativas y de movimiento, junto con verbales en un grupo multifamiliar con adolescentes con cuadros severos de anorexia. Realizan talleres de arte, grupos de discusión y también incluyen comidas multifamiliares.

\section{Focos de desarrollo del Abordaje Multifamiliar en TCA}

Según Brinchmann et al. (2017) el abordaje multifamiliar en pacientes adultos con TCA tiene cuatro focos de desarrollo:

Londres. Por parte del equipo de la Dra. Treasure en el Hospital de Maudsley (Treassure et al, 2007; Treasure, Whitaker, Todd, Whitney, 2012) desarrollan talleres educativos combinando estrategias cognitivo conductuales y entrevistas motivacionales para familias, con el fin de reducir la clínica ansiosa y depresiva de los cuidadores, el nivel de emoción expresada y los factores mantenedores del TCA relacionados con la familia, y mejorar la comunicación familiar.

Canadá. El equipo de Dimitropoulos del Hospital General de Toronto compara la terapia unifamiliar y la multifamiliar, y concluyen que la multifamiliar tuvo efectos temporales significativos para el 
IMC de los pacientes, la psicopatología relacionada con el trastorno alimentario y diversas medidas de resultados familiares. Tras un periodo de seguimiento de 3 meses no observan diferencias en ambas modalidades de tratamiento y concluyen que el grupo multifamiliar puede ser una intervención adecuada en el tratamiento de adultos con TCA. (Dimitropoulos, Freeman, Bellai y Olmsted, 2013; Dimitropoulos, Farquhar, Freeman, Colton y Olmsted, 2015).

EE.UU. En el Centro Regional para Trastornos de la Conducta Alimentaria se establece una unidad de tratamiento grupal multifamiliar en TCA que integra aspectos psicoeducativos, enfoque relacional/cultural, enfoques cognitivo conductuales y motivacionales como medio para lograr la empatía mutua y el empoderamiento, además de una conexión fuerte que permita detectar los factores mantenedores del TCA y lograr nuevos modos de relación que favorezcan la recuperación. (Tantillo, 2006; Tantillo, McGraw, Hauenstein y Groth, 2015).

Noruega. En Nordland Hospital en Bodo, el equipo de Balmbra, ValvikMand y Lyngmo (citado por Brinchmann et al., 2017) elaboran un modelo de intervención que está protocolizado y que incluye un manual explicativo del procedimiento.

Además de los mencionados ha habido otros autores que han recurrido a la utilización de grupos multifamiliares, desde orientaciones diversas, unos más desde planteamientos psicoeducativos y otros más sistémicos.

Wierenga et al. (2017) plantean un tratamiento intensivo de 5 días basado en el tratamiento multifamiliar neurobiológicamente informado para adultos con AN y sus familias. Este tratamiento centrado en el temperamento combina la psicoeducación de la neurobiología de la AN y el desarrollo de habilidades para manejar los aspectos que contribuyen a la cronicidad de la enfermedad.

En grupos multifamiliares en población adulta con TCA, el objetivo no se coloca tanto en la comida (como adultos deben asumir esta responsabilidad en nombre propio) sino en cómo avanzar hacia la recuperación o mejoría y hacia la independencia y la separación de su familia de origen (Brinchmann et al., 2017).

Algunos de los beneficios de estos grupos para familias de personas con TCA según Eisler et al. (2016) serían: favorecer el apoyo mutuo y la solidaridad, la maximización de recursos familiares, reducen la estigmatización y el aislamiento, permiten ensayar nuevas habilidades en un contexto seguro, permiten obtener una retroalimentación respecto al modo de actuar e incrementar la esperanza en la recuperación. Estos autores junto con Hughes (2018) añaden que los grupos multifamiliares incrementan la satisfacción con el tratamiento, disminuyen los abandonos, contribuyen a la mejoría del estado anímico y de la sintomatología alimentaria y disminuyen las visitas a urgencias y los reingresos hospitalarios.

Estos grupos ayudan a contener la angustia de los familiares y a descubrir formas más sanas de relacionarse con la persona con TCA (Santos, Leonidas y de Souza, 2016). A través de la creación de perspectivas múltiples y buscando activamente soluciones en lugar de confiar en expertos, las familias se empoderan y el cambio es posible (Kelly, Joyce y Lily, 2018).

Brinchmann et al. (2017) y Jewell, Blessitt, Stewart, Simic y Eisler (2016) hacen una revisión de diferentes estudios que avalan la eficacia de los grupos multifamiliares en TCA, tanto en población infantil como adulta, si bien en población adulta muchos estudios son de naturaleza cualitativa.

Algunas conclusiones provisionales que sacan respecto a los grupos multifamiliares en TCA Gelin, Fuso et al. (2015), teniendo en cuenta las limitaciones metodológicas de los estudios revisados, son: 1) que los adolescentes con AN pueden beneficiarse más que los adultos de esta modalidad de intervención, 2) que estos grupos pueden ser más eficientes o más rentables en casos resistentes al tratamiento, 3) tanto los profesionales como las familias pueden considerar estos grupos como un recurso terapéutico valioso.

\section{Nuestra Experiencia}

Nuestro contexto de trabajo es la Unidad de Trastornos Alimentarios del Hospital Universitario Santa Cristina de Madrid (UTCA), que atiende a pacientes mayores de edad procedentes de toda la Comunidad Autónoma en régimen de hospital de día. El rango de edades de las personas atendidas está entre los 18 y los 57 años en el momento actual. 
El hospital de día tiene 25 plazas en horario de mañana, donde se atiende en mayor medida un perfil de anorexia nerviosa, y otras 25 en horario de tarde para pacientes con bulimia o trastorno por atracón. Además se atienden pacientes en consulta externa, bien como paso previo a su incorporación al hospital de día o como tránsito hasta la derivación a otros dispositivos de segundo nivel. La intervención que se brinda es multidisciplinar y multidimensional, abarcando tanto los aspectos médico-psiquiátricos como los nutricionales y psicológicos. Se combinan las intervenciones individuales, grupales y familiares. Dentro de las intervenciones familiares se incluyen la entrevista familiar al comienzo de tratamiento, los grupos de padres, grupos multifamiliares y la terapia de familia en aquellos casos donde los factores familiares actúan como claros mantenedores del TCA.

Damos mucha importancia al estadio motivacional en el que se encuentren los pacientes y las familias de cara a organizar las intervenciones, y al establecimiento de un vínculo terapéutico seguro tanto con el paciente como con los familiares. Gran parte de nuestro trabajo en el hospital de día sucede en el aquí y ahora de las interacciones que se dan entre los pacientes, y entre éstos y el equipo, reproduciéndose modos de funcionar similares a los adoptados en la familia y en otros contextos. Este espacio brinda la oportunidad de numerosas experiencias emocionales correctivas y de desarrollar nuevos modos de interactuar y de mejorar las habilidades relacionales.

Desde que se creó el servicio en el año 2006 se venían realizando grupos de padres y parejas por parte del equipo de la mañana que, como se ha dicho, trata más el perfil de anorexia nerviosa. En el año 2011 se introduce de forma complementaria el abordaje multifamiliar, en un principio, con periodicidad mensual. En la actualidad, de cara a facilitar la participación de las familias y también por razones de espacio, se llevan a cabo dos grupos, uno los miércoles a primera hora y otro los viernes a media mañana. La media de participación es de unas 7 u 8 familias por grupo. La periodicidad es quincenal y la asistencia es flexible (pueden venir indistintamente a cualquiera de los dos espacios grupales). En ambos grupos participan tanto los pacientes y familiares de las personas que están ingresadas en la UTCA como aquellos que ya están en régimen de consulta externa. Además, desde hace seis meses se incluyen otras personas significativas del contexto social del paciente (p.e. amigos). La codirección la realizan la psiquiatra y la psicóloga clínica. También está abierto a otros profesionales, de modo habitual participan el terapeuta ocupacional, los residentes de psicología, psiquiatría y enfermería que rotan por la unidad y los estudiantes del Máster de Trastornos de la Alimentación de la Universidad Europea y del Máster de Psicología General Sanitaria de la Universidad Autónoma.

Después de cada grupo, el equipo se reúne para revisar lo sucedido, se ponen en común las diferentes visiones de los profesionales participantes extrayendo temáticas significativas para abordar en otros espacios terapéuticos o en el siguiente grupo multifamiliar. Este post-grupo también sirve para detectar si es oportuno realizar alguna sesión de terapia familiar para trabajar algún aspecto que consideramos que está actuando como factor mantenedor del TCA.

Desde el punto de vista teórico, nuestra filosofía encaja con el planteamiento de la Terapia Interfamiliar de Sempere y Fuenzalida (2017) que, como ya hemos referido en apartados anteriores, es un modelo integrador que combina planteamientos sistémicos y de la terapia familiar (en nuestro caso fundamentalmente desde la escuela estructural, estratégica y también teniendo en cuenta los aspectos transgeneracionales), con conceptos provenientes de la teoría del apego (importancia de los vínculos y del establecimiento de una base segura, el impacto del trauma relacional, las consecuencias de la inseguridad en el apego, etc.), conceptos de la psicología dinámica (mentalización, mecanismos de defensa, el cambio y la construcción de la identidad a través de la interacción con los demás, etc.), de la terapia de grupo y del enfoque de ayuda mutua e interaccional, el diálogo reflexivo, y del modelo de comunidad terapéutica (Sempere y Fuenzalida, 2013).

Al igual que Colahan y Robinson (2002), además de recursos verbales también incluimos otras técnicas de carácter no verbal o artístico, por ejemplo el trabajo con fotografías de modelos de familia o las esculturas familiares (la real y la deseada) realizadas por los diferentes miembros de la familia, favoreciendo la reflexión acerca de las similitudes y diferencias existentes entre las mismas. También se incluyen otros recursos como los cuentos, pinturas, metáforas y escenas de películas sobre temas relacionados con las dinámicas familiares, los modos comunicacionales, secretos familiares, y otras temáticas que surgen. 
Por otro lado, teniendo en cuenta la gravedad de la sintomatología de las pacientes que tratamos, incluimos también elementos psicoeducativos, herramientas provenientes de la terapia cognitivo-conductual (análisis funcional, leyes del aprendizaje, técnicas como exposición y prevención de respuesta, manejo de contingencias, etc.), y de las Terapias de Tercera Generación, fundamentalmente de la Terapia de Aceptación y Compromiso, de la que tomamos conceptos como la tolerancia al malestar, la importancia de los valores personales, y la evitación experiencial entre otros.

Desde el punto de vista técnico, los terapeutas adoptan un modelo flexible, favoreciendo el diálogo entre los participantes, pero interviniendo en momentos puntuales para reconducir el grupo, romper escaladas simétricas, limitar a miembros que acaparan demasiado el grupo o que lo llevan a "hablar de la nada" y para rescatar a algún miembro que está siendo excesivamente cuestionado. Damos mucha importancia a la espontaneidad y al uso del sentido del humor como forma de mantener un nivel de arousal adecuado, dado que tanto si es muy bajo como si es muy elevado dificulta la capacidad de análisis e integración de lo trabajado.

\section{Temáticas de los Grupos}

Las temáticas que surgen, en ocasiones de forma espontánea y en otras planteadas por algún miembro del grupo o del equipo, son muy variadas, de hecho en un solo grupo se pueden trabajar muchos aspectos a la vez. A continuación expondremos algunas de ellas.

Dada la larga trayectoria de enfermedad que tienen la mayoría de los pacientes atendidos en la UTCA, un patrón bastante generalizado que se observa es que las interacciones familiares están centradas en el TCA, quedando difuminados los aspectos positivos y sanos que tienen los pacientes y también el resto de los miembros de la familia. Un trabajo fundamental que facilitan los grupos multifamiliares es lograr un cambio en la mirada, y girar el foco hacía estos aspectos que son observados por otros miembros del grupo y que pasan desapercibidos para la propia familia.

Los familiares que acuden por primera vez a los grupos vienen cargados con la angustia, la impotencia y la frustración de haber visto a su hijo/a durante años poner en riesgo su vida. Inicialmente sus intervenciones están focalizadas en el tema de la comida y el peso. En este caso, el grupo brinda apoyo emocional a estos miembros, les transmite que el problema va mucho más allá de la comida o el peso, y de modo paulatino les reconduce para hablar de otras cuestiones.

En ocasiones, el grupo también permite evidenciar y tomar conciencia de patrones alimentarios disfuncionales en otros miembros de la familia.

En cuanto a la posición de los padres o familiares respecto al TCA observamos dos posturas, por un lado algunos padres vienen cargados de un sentimiento de culpa respecto al problema del hijo, y en otras ocasiones adoptan una postura absolutamente externalizadora responsabilizando a otras personas ajenas al ámbito familiar (parejas, profesores, entrenadores, etc.) o al propio paciente, planteando: "nosotros no tenemos nada que ver en el problema". En algunos casos, la actitud sostenida en relación al hijo/a ha sido de carácter sobreprotector y en otros -generalmente los casos más graves- han adoptado una postura emocionalmente negligente. Algunas de las intervenciones grupales van orientadas a plantear que lo importante no es buscar culpables sino soluciones, y que en ocasiones desde la buena intención se pueden realizar acciones que causan daño al otro, y es importante poder hablarlas para dar la oportunidad de reparar y rectificar.

A lo largo del funcionamiento del grupo se ponen de manifiesto cuestiones referentes a la estructura y al funcionamiento familiar: cambios de roles (hijos cuidadores de los padres o sustitutos de parejas, hermanos parentalizados, etc.), triangulación en conflictos de pareja, alianzas entre miembros o coaliciones de unos contra otros, miembros en posición periférica, aglutinamiento o dificultad para funcionar como sujeto diferenciado (abuso del nosotros, "somos una piña", el discrepar vivido como traición), problemas de diferenciación de los padres respecto a sus familias de origen e intromisiones de la familia extensa con dificultades para limitarlas (en muchas ocasiones se usa el TCA de la hija para limitar las visitas no deseadas). También se observan normas no acordes con el momento evolutivo ( $\mathrm{Ej}$. adultas/os que tienen que llamar varias veces al día a su madre/padre para darle "el parte de lo que hace"), dificultades comunicacionales e interacciones repetitivas 
de carácter patológico. A través del aprendizaje vicario, de las interacciones entre los diferentes miembros del grupo y de los señalamientos mutuos, se abordan estos aspectos ayudando a reconstruir narrativas y adoptar perspectivas alternativas en las interacciones establecidas y respecto a los acontecimientos familiares vividos.

Otro tema que aparece con frecuencia en estos grupos es la necesidad de ajustar las expectativas, tanto de lo que espero de mí como lo que espero del otro, y ayudar a desligar los deseos propios de los deseos de los demás. En muchas ocasiones los pacientes se han limitado a seguir el camino marcado a costa de renunciar a ser ellos mismos. Pero también es importante que los hijos ajusten las expectativas respecto a lo que pueden esperar o no de los padres siguiendo el lema "no pidas peras al olmo".

Desde nuestra conceptualización del tratamiento, la toma de decisiones es una parte esencial del proceso de recuperación, así como la definición de los errores como oportunidades para el aprendizaje. Coincidimos con los preceptos de la Terapia de Aceptación y Compromiso (Wilson y Luciano, 2002) de que la AN de larga evolución es un trastorno de evitación experiencial. Por esta razón, dos de los lemas del funcionamiento del grupo multifamiliar (y de la UTCA en general) son "no le des peces, enséñale a pescar" y "mejor vivir aún a riesgo de errar, que mantenerse en un permanente vacío existencial". Por todo esto, en el grupo no se trata tanto de dar soluciones o consejos (aunque se puede hacer), sino de hablar desde las propias experiencias abriendo alternativas diferentes para que sea la persona, la que en última instancia decida en base a sus valores y al sentido de su vida lo que considere más pertinente. Por esta razón, en el grupo se trabajan mucho todas las cuestiones existenciales como la falta de vida propia, la carencia de objetivos vitales, la insatisfacción vital, las pérdidas, etc. Estos temas no afectan solo a los pacientes identificados sino también a las familias.

Otra temática recurrente en los grupos es la dificultad que tienen los miembros (tanto pacientes como familiares) para aceptar el malestar y las emociones negativas tanto propias como ajenas. Algunas frases que se repiten con mucha frecuencia es "no le digo las cosas porque no quiero hacerle daño", "de eso no se habla porque duele" y otras similares. Estas expresiones ponen de manifiesto que por temor a herir, molestar o enfadar al otro, al final las relaciones se convierten en algo banal, falso o vacío de contenido. A través de las interacciones propias y observadas, y con el apoyo del espacio grupal, se ponen en cuestión estos planteamientos favoreciendo la creación de relaciones más genuinas entre los miembros.

Cuando el grupo es vivido como un lugar seguro y de confianza, con frecuencia salen a relucir vivencias traumáticas, tanto de los pacientes como de los familiares y numerosos secretos familiares que han obstaculizado el acercamiento emocional entre los miembros. En estos casos el acogimiento emocional que brinda el grupo es fundamental para lograr una experiencia emocional correctiva.

De cara a valorar la impresión de los pacientes y de las familias acerca del grupo multifamiliar, realizamos una breve encuesta acerca de qué ventajas y qué inconvenientes les atribuyen a estos grupos, solicitando un consentimiento informado para utilizar de forma anónima sus respuestas. Los datos obtenidos figuran en las tablas 1 y 2 .

Además del grupo multifamiliar y en semanas alternas, mantenemos un grupo de padres. Consideramos que ambas intervenciones no son excluyentes sino complementarias, y que de este modo se transmite de forma simbólica que hay aspectos tanto de los pacientes como de las familias que no siempre se comparten con el resto de los miembros al formar parte del espacio privado o íntimo. Al igual que los pacientes disponen de diferentes espacios grupales en el hospital de día para trabajar aspectos de su vida, no siempre relacionados con el área familiar, es importante que los padres también dispongan de su espacio para abordar temas personales o de pareja. En este sentido no es un grupo de carácter psicoeducativo.

En ocasiones algunos temas que se tratan el grupo de padres o en grupos de pacientes pueden salir en los multifamiliares o viceversa, sin que esto suponga problema alguno sino más bien un enriquecimiento en el trabajo grupal.

Cuando la conflictiva familiar es muy intensa o se sostienen unos patrones de funcionamiento rígidos que favorecen el mantenimiento de la patología alimentaria y obstaculizan el desarrollo individual de los miembros, se recurre a llevar a cabo un número cerrado de sesiones de terapia familiar. Además, cuando se observa patología en alguno de los familiares, se les sugiere la búsqueda de tratamiento para ellos. Algunos de los trastornos que observamos con frecuencia en familiares son cuadros ansioso-depresivos, trastornos de la alimentación, alcoholismo, trastornos obsesivo-compulsivos y rasgos disfuncionales de la personalidad entre otros. 


\section{Tabla 1: Impresiones de los padres/familias}

\section{Las ventajas de estos grupos serían:}

- "El grupo me permite decir cosas que en casa no me atrevería, o afrontar temas que son difíciles".

- "Ayuda a saber que no somos los únicos que les pasa este problema".

- "Favorece la escucha mutua" y "Ayuda a afrontar dificultades".

- "Ayuda a ver que no somos perfectos...tampoco los demás...todos nos equivocamos",

"nos ayuda a tener una visión realista, no somos tan raros".

- "Te permiten ver aspectos tuyos y de tu hija que habían pasado desapercibidos".

- "Te ayudan a ampliar la perspectiva... a reflexionar sobre cosas que en el día a día no te dabas cuenta".

- "Ayuda a vencer tabúes para hablar de tus cosas al observar que otros también lo hacen... sin sentirte juzgado... sacas temas que igual tu solo no sacarías".

- "Ayuda a dar importancia a unas cosas y a relativizar otras no tan graves".

- "Ayudan a modificar actitudes y comportamientos disfuncionales".

- "Es una ayuda para mi...para comprender mejor las relaciones humanas", "te sientes apoyado".

- "Al principio lo veía como una obligación: "buen padre, implicado"...ahora me doy cuenta de que también me aporta a mí en lo personal".

- "Somos parte de la solución porque somos parte del problema...éste nos afecta a todos".

- "No sirven para curar a tu hijo. Sirven para curar heridas personales haciéndote mejorar como persona a todos los niveles".

- "He aprendido a ser madre sin miedo a decir lo que pienso, no solo con ella, también con los demás...he crecido como persona".

- "Me ha ayudado a quererme más y a querer más a los demás".

\section{Los inconvenientes:}

- "Da vergüenza hablar en público".

- "Cuesta sacar en grupo los trapos sucios".

- "Es duro saber que la terapia es lenta y los cambios se producen más despacio de lo que te gustaría".

\section{Tabla 2. Impresiones de los pacientes}

\section{Ventajas de los grupos:}

- "Ver experiencias similares ayuda a enriquecer las tuyas".

- "Dices cosas que en casa no te atreverías".

- "Ayuda a la escucha activa y al diálogo más sosegado que en casa".

- "Ayuda a que dejen de verte como un bicho raro".

- "Ayuda a entender cosas de los padres respecto a sus familias de origen".

- "Ayudan a empatizar con los padres y a no verles como los enemigos, sino como aliados".

- "Ayudan a aumentar la comprensión y apoyo mutuo y la cercanía emocional"

- "Ayudan a afrontar problemas en vez de evitarlos".

- "A veces pueden originarse conflictos o tensiones pero es mejor que vivir en la ignorancia".

- "Ayudan a empatizar unos con otros".

- "Son útiles aunque no esté tu familia porque ver las interacciones entre otros padres e hijos o ver a otros padres ayuda a resolver cosas con los tuyos". 


\section{Tabla 2 (Continuación). Impresiones de los pacientes}

- "Me ayuda como paciente a entender cosas de mis padres y, como madre a cambiar y mejorar cosas en relación con mis hijos".

- "A veces te ayuda verbalizar a otro padre/madre cosas que no te atreves a decir al tuyo/a".

- "Tengo que aceptar, al faltar la implicación de mis padres, que no me queda otra que tirar sola".

- "Se pone de manifiesto que hay roles que están cambiados".

- "Ayudan a pedir perdón y a reparar".

\section{Inconvenientes:}

- "Sentimientos de culpa porque tienen que faltar a sus ocupaciones por venir".

- "Es duro que te confirmen ciertas realidades que tu percibías pero no querías aceptar...bueno esto tiene un lado positivo también y es saber que tu idea no era equivocada"..."ayuda a caerte del guindo".

- "Los padres pueden echarte en cara en casa lo que has hablado".

- "Los míos acuden pero no hacen cambios...me piden que cambie yo".

\section{Conclusiones}

Los grupos multifamiliares se integran como una herramienta más en el engranaje complejo que supone tratar patología mental grave en el contexto de hospital de día.

Las complicaciones físicas y psicológicas de los pacientes tratados exigen una combinación de intervenciones médicas, nutricionales y psicológicas de índole diversa. La experiencia profesional de 20 años tratando TCA de larga evolución nos ha orientado hacia un modelo de tratamiento integrado e integrador, al no haber encontrado un paradigma teórico que por sí solo y de forma exclusiva de explicación a toda la complejidad de la realidad humana y permita abordar una patología tan compleja como los TCA. En este sentido, somos un equipo en permanente evolución y formación, que rechaza las posturas reduccionistas que suponen la adscripción rígida a un único modelo teórico. Estamos abiertos a aprender, no solo de otros profesionales sino también y sobre todo, de nuestros pacientes y familias.

La puesta en marcha del grupo multifamiliar en la UTCA surge de la necesidad observada, tanto en las familias como en los pacientes, de facilitar un espacio de interacción y apoyo del que no siempre disponen en sus casas. Y también, como un modo de incrementar la eficiencia del equipo, ahorrando costes y tiempo en el tratamiento.

El paradigma desde el que manejamos estos grupos es de carácter integrador y se aproxima mucho a la Terapia Interfamiliar de Sempere y Fuenzalida (2017).

Si bien no hemos realizado estudios de carácter cuantitativo que avalen nuestra experiencia, consideramos que son grupos de gran potencialidad terapéutica. Desde su puesta en funcionamiento, venimos observando una serie de indicadores relacionados con la eficacia y efectividad de los mismos, tales como: la facilitación de la mejoría clínica de los pacientes, la reducción del número de readmisiones en las unidades de hospitalización total por empeoramiento clínico, una disminución de las visitas a urgencias de las pacientes, un aumento de la satisfacción en pacientes y familias, y una reducción de los abandonos del tratamiento. Por otro lado, observamos que aquellos pacientes cuyas familias no están implicadas en el tratamiento tienen una peor evolución clínica. Estas impresiones coinciden con las referidas en la literatura y con las experiencias de compañeros de otros dispositivos que también realizan grupos multifamiliares, con los que compartimos impresiones y supervisamos.

Es importante recalcar de nuevo que estos grupos se llevan a cabo en el contexto de hospital de día, donde se incluyen otras intervenciones terapéuticas, además de los efectos propios de la convivencia entre pacientes y equipo. Por ello, los efectos terapéuticos no se limitan exclusivamente a la realización de estos grupos. 
Sería necesario realizar una investigación que tenga en cuenta las distintas variables y realice comparaciones con un grupo control, para poder formular relaciones de causalidad que avalen nuestras impresiones.

\section{Referencias}

Abrahams, J. y Varon, E. (1953). Maternal Dependency and Schizophrenia: Mothers and patiens in a Therapeutic Group. A group analytic study. New York, Estados Unidos: International University Press.

Alcalde, A. y Montero, M. T. (2014). Una Experiencia de Grupo Multifamiliar con personas con Trastorno Mental Grave. Clínica Contemporánea, 5, 205-212. https://doi.org/10.5093/cc2014a14

Asen, E. (2002). Multiple family therapy: an overview. Journal of Family Therapy, 24, 3-16.

Asen, E. (2006). Systemic approaches-critique and scope. En S. Timimi and M. Begum (Eds), Critical Voices in Child and Adolescent Mental Health. Londres, Reino Unido: Free Association Books.

Arroyo Guillamón, R., Fernández-Mayoralas Rubio, V., Reguera Nieto, E. y Martínez Pérez, C. (2014). Psicoanálisis multifamiliar: una experiencia formativa grupal. En A. Ceverino Domínguez, (Coord.), Salud mental y terapia grupal. Madrid, España: Grupo 5. Recuperado de https://www.aacademica.org/rafael.arroyo.guillamon/6.pdf

Ayerra, J. M. y López, J. L. (2003). El grupo multifamiliar: un espacio sociomental. Avances en Salud Mental Relacional, 2(1). Recuperado de https://studylib.es/doc/8321327/el-grupo-multifamiliar--un-espacio-sociomental. Bion, W. R. (1985). Experiencias en grupos. Barcelona, España: Paidós.

Blajakis, M. I. y López Atienza, J. L. (2002). Tratamiento multigrupal de la adolescencia (integración de grupo pequeño y de grupo multifamiliar). Boletín 27, 3-14. Recuperado de http://www.fundacioorienta.com/ wp-content/uploads/2019/03/Sempere-Javier-5.pdf

Bowen, M. (2014). De la familia al individuo. La diferenciación del sí mismo en el sistema familiar. Barcelona, España: Paidós.

Brinchmann, B. S. Moe, C., Valvik M. E. Balmbra, S., Lyngmo, S., Lyngmo, S. y Skarbo, T. (2017). An Aristotelian view of therapists' practice in multifamily therapy for young adults with severe eating disorders. Nursing Ethics, 26(4), 1149-1159. https://doi.org/10.1177\%2F0969733017739780

Burguillo, F. (2010). La magia del psicoanálisis multifamiliar. La articulación del modelo psicoanalítico con la realidad del trabajo en el sistema público. Avances en Salud Mental Relacional, 9(2), 1-14.

Canzio, C. y Zurkirch, V. (2015). Grupos multifamiliares desde el Psicoanálisis integrativo. Experiencia de sostén donde se encuentran grupos familiares y grupos institucionales en el servicio público de la ciudad de Florencia. Área 3. Cuadernos de Temas Grupales e Institucionales, 19. Recuperado de http://www.area3.org. es/uploads/a3-19-GruposMultifamiliares-CCanzio-VZurkirch_1.pdf

Cook-Darzens, S. (2014). The role of family meals in the treatment of eating disorders: a scoping review of the literature and implications. Eating and Weight Disorders, 21,383-39. https://doi.org/10.1007/s40519-016-0263-y

Cooklin, A., Miller, A. y McHugh, B. (1983). An institution for change: developing a family day unit. Family Process Journal, 22, 453-468.

Colahan, M. y Robinson, P.H., (2002). Multi-family groups in the treatment of young adults with eating disorders. Journal of Family Therapy, 24, 17-30. https://doi.org/10.1111/1467-6427.00198

Conner, K. R, Shea, R. R., McDermott, M. P, Grolling, R., Tocco, R. V. y Baciewicz, G. (1998). The Role of Multifamily Therapy in Promoting Retention in Treatment of Alcohol and Cocaine Dependence. The American Journal on Addictions,7(1), 61-73.

Dare, C. y Eisler, I. (2000). A multifamily group day treatment programme for adolescent eating disorder. European Eating Disorders Review, 8(1), 4-18.

De Maré, P. (1988). La historia del grupo grande y sus fenómenos en relación a la psicoterapia de grupo analítica. Barcelona, España: Ediciones Plexus.

Dimitropoulos, G., Freeman, V. E., Bellai, K. y Olmsted, M. P. (2013). Inpatients with severe anorexia nervosa and their siblings: non-shared experiences and family functioning. European Eating Disorders Review, 21(4), 284-293. https://doi.org/10.1002/erv.2230 
Dimitropoulos, G., Farquhar, J. C., Freeman, V. E., Colton, P. A. y Olmsted M. P. (2015). Pilot study comparing multi-family therapy to single family therapy for adults with anorexia nervosa in an intensive eating disorder program. European Eating Disorders, 23, 294-303. https://doi.org/10.1002/erv.2359

Edwards S. (2001). The essential elements of multi-family group therapy: a Delphi study (Tesis doctoral). Faculty of the Virginia Polytechnic Institute and State University, Estados Unidos.

Eisler, I., Simic, M., Hodsoll, J., Asen, E., Berelowitz, M., Connan. F... Landau, S. (2016). A pragmatic randomised multi-centre trial of multifamily and single family therapy for adolescent anorexia nervosa. $B M C$ Psychiatry, 16, 422. https://doi.org/10.1186/s12888-016-1129-6

Fortunato, L. F., Ribeiro, A., Lôbo, E., Figueiredo, F. y Cavalcanti, L. M. (2011). Grupo multifamiliar com adolescentes ofensores sexuais. Psico, 42, 450-456.

Foster, L. N. (1991). A theoretical framework for multiple family group therapy with chemically dependent and other at-risk families. Recuperado de http://www.multiplefamilygrouptherapy.com/The\%20Foster\%20 Model\%20of\%20MFGT\%20With\%20CD\%20Families.htm

Foulkes, S. H. (1984). Therapeutic group analysis. Londres, Reino Unido: Maresfield.

García Badaracco, J. (1989). Comunidad terapéutica psicoanalítica de estructura multifamiliar. Buenos Aires, Argentina: Tecnipublicaciones.

García Badaracco, J. (2000). Psicoanálisis Multifamiliar. Los otros en nosotros y el descubrimiento del sí-mismo. Buenos Aires, Argentina: Paidós.

Gelin, Z., Cook-Darzens, S., Simon, Y. y Hendrick, S. (2015). Two models of multiple family therapy in the treatment of adolescent anorexia nervosa: a systematic review. Eating and Weight Disorder, 21, 19-33. https://doi.org/10.1007/s40519-015-0207-y

Gelin Z., Fuso S., Hendrick S., Cook-Darzens S. y Simon Y. (2015). The effects of a multiple family therapy on adolescents with eating disorders: an outcome study. Family Process Journal, 54(1), 160-172. https://doi. org/10.1111/famp.12103

Gergen, K. J. (1973). Social Psychology as History. Journal of Personality and Social. Psychology. 26(2), 309-320.

Gulliksen, K. S., Espeset, E. M. S., Nordbø, R. H. S. y Holte, A. (2012). The link between negative emotions and eating disorder behaviour in patients with anorexia nervosa. European Eating Disorders Review, 20, 451-60. https://doi.org/10.1002/erv.2183

Hughes, E. K., (2018). Multifamily therapy may add to the effectiveness of single-family therapy for adolescents with anorexia nervosa. Evidence-Based Mental Health, 21, e4. http:// doi.org/10.1136/eb-2017-102732

Jewell, T., Blessit, E., Stewart, C., Simic, M. y Eisler, I. (2016). Family Therapy for Child and Adolescent Eating Disorders: A Critical Review. Family Process Journal, 55, 577-594.

Kaufman,E. (1977). Multifamily Therapy: a new direction in the treatment of drug abusers. American Journal of Drug and Alcohol Abuse, 4, 467-478.

Kelly, Y. C., Joyce, L. C. y Lily, L. L. (2018). Multifamily Therapy for Children with ADHD in Hong Kong: The Different Impacts on Fathers and Mothers. Journal of Attention Disorders. https://doi. org/10.1177/1087054718756195

Laqueur, H. P. (1976). Multiple family therapy. En P. J. Guerin (Ed.), Family therapy, theory, and practice (pp. 405-416). Nueva York, Estados Unidos: Gardner.

Lock, J., Le Grange, D., Agras, W. S. y Dare, C. (2001). Treatment manual for anorexia nervosa: A family-based approach. Nueva York, Estados Unidos : Guilford Press.

McNamee, S. y Gergen, K. J. (1996). Terapia como construcción social. Barcelona, España: Paidós.

Martínez, M. y Perles, F. (2010). Experiencias en grupos multifamiliares en el hospital de día de la Axarquía (Vélez Málaga). Avances en Salud Mental Relacional, 9(2). Recuperado de https://psiquiatria.com/bibliopsiquis/experiencias-en-grupos-multifamiliares-en-el-hospital-de-dia-de-la-axarquia-velez-malaga/

Mascaró, N. (2007). Crisis y contención: El grupo multifamiliar. Avances en Salud Mental Relacional, 6(2), 1-9. Mascaró, N. (2010). García Badaracco y la Terapia familiar. Avances en Salud Mental Relacional, 9(1), 1-6.

Mc Farlane, W. R. (2002). Multifamily Groups in the Treatment of Severe Psychiatric Disorders. Nueva York, Estados Unidos: Guilford Press. 
Pérez, M., Jiménez J. A., Torregrosa, S. y Sempere, J. (febrero, 2016). Terapia grupal multifamiliar en el abordaje de la problemática conductual y emocional del adolescente. Trabajo presentado en el $17^{\circ}$ Congreso Virtual de Psiquiatria.com, Interpsiquis. Resumen recuperado de http://psiqu.com/1-5691

Pichón-Rivière, E. (1985). El proceso grupal: del psicoanálisis a la psicología social. Buenos Aires, Argentina: Nueva Visión.

Ramos, L. y de Miguel, S. (2017). Grupo multifamiliar en un hospital de día de trastornos de la conducta alimentaria. Avances en Salud Mental Relacional, 16(3), 1-18.

Rienecke, R. D. (2017). Family-based treatment of eating disorders in adolescents: current insights. Adolescent Health, Medicine and Therapeutics, 8, 69-79.

Santos M.A., Leonidas, C. y de Souza L. R. (2016). Grupo multifamiliar no contexto dos Transtornos Alimentares: a experiência compartilhada. Arquivos Brasileiros de Psicología, 68(3), 43-58.

Saunders, T. (1990). Go ahead, kill yourself! Save your family trouble: paradoxical therapy with families. Plantation, Estados Unidos: Distinctive Publishing.

Scholz, M. y Asen, E. (2001). Multiple family therapy with eating disordered adolescents: concepts and preliminary results. European Eating Disorders Review, 9, 33-42.

Seikkula, J. (1994). When the boundary opens: Family and hospital in co-evolution. Journal of Family Therapy, 16, 401-414.

Sempere, J. (2012). Nexos del psicoanálisis multifamiliar con otros enfoques terapéuticos. Consideraciones acerca de una nueva epistemología. Teoría y práctica grupoanalítica, 2(1), 11-20.

Sempere, J. y Fuenzalida, C. (2013). Terapia interfamiliar: de la "terapia de familia" a la "terapia entre familias”. Revista Psicoterapia y Psicodrama, 2(1), 88-105

Sempere, J. y Fuenzalida, C. (2017). Terapias multifamiliares. El modelo interfamiliar: La terapia hecha entre todos. Madrid, España: Psimática Editorial.

Slagerman, M. y Yager, J. (1989). Multiple family group treatment for eating disorders: A short term program. Psychiatric Medicine, 7, 269-283.

Steinglass, P. (1998). Multiple family discussion groups for patients with chronic illness: Families, Systems and Health, 16, 55-70.

Stewart, C., Voulgari, S., Eisler, I., Hunt, K. y Simic, M. (2015). Multi-family therapy for bulimia nervosa in adolescence. Eating Disorders, 23(4), 345-355. https://doi.org/10.1080/10640266.2015.1044348

Sullivan, H. (1953). The interpersonal theory of psychiatry. Nueva York, Estados Unidos: Norton.

Tantillo, M. (2006). A relational approach to eating disorders multifamily therapy group: Moving from difference and disconnection to mutual connection. Families, Systems, \& Health, 24(1), 82-102. https://doi. org/10.1037/1091-7527.24.1.82

Tantillo, M., McGraw, J. S., Hauenstein, E. J. y Groth, S. W. (2015). Partnering with patients and families to develop an innovative multifamily therapy group treatment for adults with anorexia nervosa. Advances Eating Disorders: Theory, Research and Practice,3(3), 269-287. https://doi.org/10.1080/21662630.2015.1 048478.

Treassure, J. Smith, G. y Crane, A. (2007). The clinician's guide to collaborative caring in eating disorders: The New Maudsley method. Londres, Reino Unido: Routledge.

Treasure, J., Whitaker, W., Todd, G. y Whitney, J. (2012). A description of multiple family workshops for careers of people with anorexia nervosa. European Eating Disorders Review, 20, 17-22. https://doi.org/10.1002/ $\underline{\text { erv. } 1075}$

Uría, T., Ahijado, Z., Serrano, A. Mira, J. y Rubio A. (marzo, 2016). El grupo multifamiliar en el hospital de día de niños. Comunicación presentada en el XXVIII Congreso de SEPYPNA, Navarra, España.

Von Bertalanffy, L. (1976). Teoría general de los sistemas. Fundamentos, desarrollo, aplicaciones. México, D. F., México: Fondo de Cultura Económica.

Watzlaswick, P., Beavin, J. y Jackson, D. D. (1995). Teoría de la comunicación humana. Interacciones, patologías y paradojas. Barcelona, España: Herder.

Wierenga, C. E., Hill, L., Knatz Peck, S., McCray, J., Greathouse, L., Peterson, D., Scott, A., Eisler, I. y Kaye, W.H. (2017). The acceptability, feasibility, and possible benefits of a neurobiologically-informed 5-day mul- 
tifamily treatment for adults with anorexia nervosa. International Journal of Eating Disorders, 51, 863-869. https://doi.org/10.1002/eat.22876

Wilson, G. K y Luciano, M. C. (2002). Terapia de aceptación y compromiso (ACT): un tratamiento conductual orientado a los valores. Madrid, España: Pirámide.

Wooley, S .C. y Lewis, K. (1987). Multi-family therapy within an intensive treatment program for bulimia. En J. Harkaway (Ed.), Eating disorders (pp. 12-24). Rockville, Estados Unidos: Aspen Publishers.

Yalom, I. D. (1995). The theory and practice of group psychotherapy (4a Edición). Nueva York, Estados Unidos: Basic Books.

Artículo recibido: 17/07/2018

Artículo aceptado: 04/06/2019 